\title{
ACOUSTIC VOICE ANALYSIS IN PATIENT WITH DYSPHAGIA
}

\author{
Howook Kim¹, Sooho Park', Youngae Kang², Bonseok Koo², Sungju Jee ${ }^{1}$
}

${ }^{1}$ Department of Rehabilitation Medicine, ${ }^{2}$ Department of Otolaryngology-Head and Neck Surgery Chungnam National University Hospital, Daejeon, Korea

\section{$\checkmark$ Introduction}

$>$ Penetration and aspiration (P/A) might result in some residue in the vocal folds and alter the voice quality.

$>$ These changes on the vocal folds will be reflected in the acoustic voice parameters.

$>$ The purpose of this study was to investigate feasibility of voice acoustic parameters on penetration / aspiration (P/A).

Subjects

$>$ September 9 to October 29, 2015. All patients were taken under videofluoroscopic swallowing study (VFSS)

- Yogurt $\rightarrow$ porridge $\rightarrow$ boiled rice $\rightarrow$ water $(5,10 \mathrm{~mL})$ with Liquid barium

Table 1. Demographic data of study patients

\begin{tabular}{lcccc}
\hline & Total & Non P/A & P/A & P value \\
$\mathrm{n}$ & 23 & 10 & 13 & \\
Sex, male / female & $15 / 8$. & $5 / 5$. & $10 / 3$. & 0.195 \\
Age (yrs.) mean \pm SD & $69 \pm 10.1$ & $71.6 \pm 8.9$ & $67.1 \pm 11$. & 0.331 \\
Cause of dysphagia & & & 1 & \\
Stroke & & 6 & 7 & \\
Medical disease & & 2 & 3 & \\
Head-neck cancer & 0 & 2 & \\
Degenerative disesase & 1 & 0 & \\
Traumatic brain Injury & 1 & 1 & \\
\hline
\end{tabular}

Method

$>$ Voice recording : a single /a/ vowel pre- \& post VFSS

- Sennheiser pc151 (KG, Germany)

- PCM-50 (sony Corp. Tokyo, Japan)

- $48 \mathrm{KHz}$ sampling rate, 26 bit depth

$>$ Acoustic analysis (using Multi-Dimensional Voice Program (MDVP))

- $\quad \mathrm{mF} 0$ (Average Fundamental Frequency of voice)

- RAP (Relative Average Perturbation )

- Shimmer (variability of the peak-to-peak amplitude )

- NHR (Number of Subharmonic Segments)

- VTI (Voice Turbulence Index )

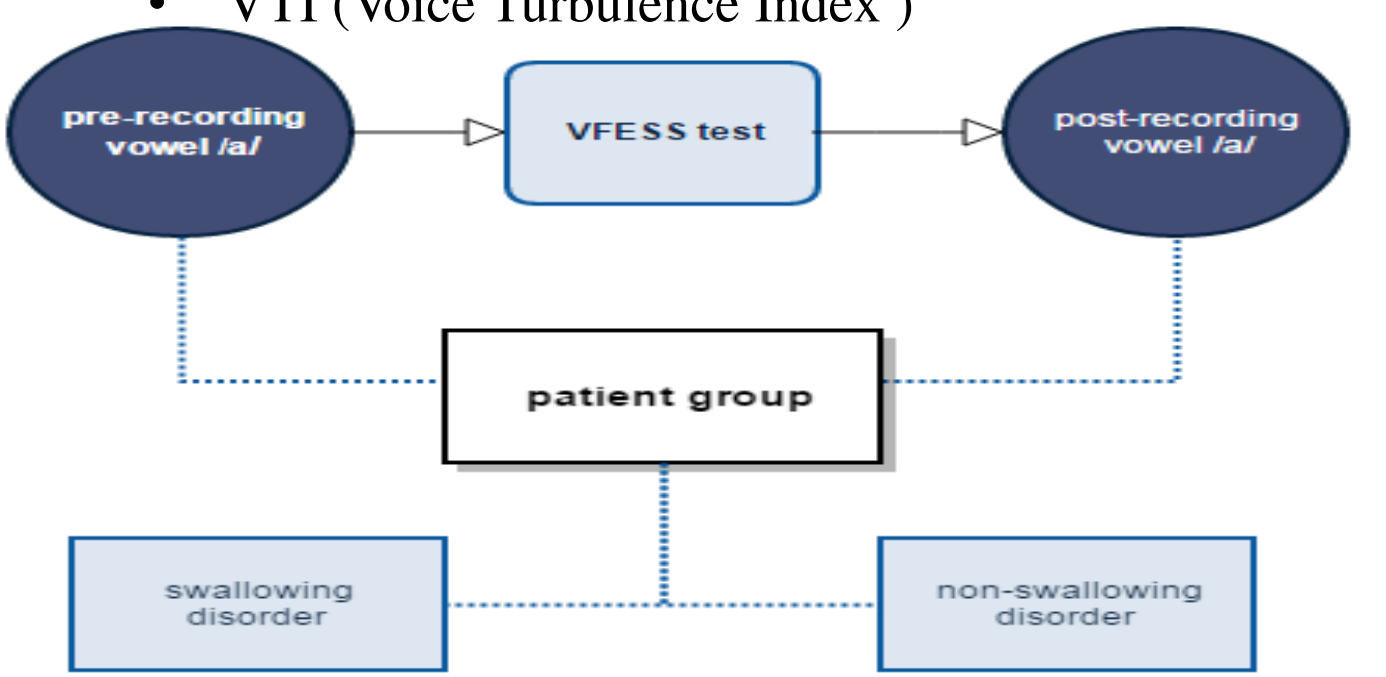

Figure1. Patients Flow Chart

Table 2. Differences in acoustic voice parameters pre- and post

\begin{tabular}{lccc}
\multicolumn{4}{c}{ VFSS } \\
Parameter & Non P/A & P/A & P value \\
mF0 (in male) & $-8.41 \pm 12.58$ & $3.54 \pm 9.92$ & 0.065 \\
& & - & \\
mF0 (in female) & $3.88 \pm 58.48$ & $11.88 \pm 50.2$ & 0.713 \\
& & 9 & \\
RAP & $1.33 \pm 2.23$ & $0.70 \pm 2.77$ & 0.584 \\
Shimmer & $5.43 \pm 7.76$ & $0.49 \pm 4.72$ & 0.072 \\
NHR & $0.13 \pm 0.17$ & $0.03 \pm 0.12$ & 0.134 \\
VTI & $0.09 \pm 0.13$ & $-0.01 \pm 0.07$ & 0.028
\end{tabular}

P/A penetration / aspiration; mFo Average Fundamental Frequency; RAP Relative Average Perturbation; NHR Noise to Harmonic Ratio; VTI Voice Turbulence Index

This could be a place for your sources.
Table 3. ROC with difference pre-and post VFSS

\begin{tabular}{lcc}
\multicolumn{1}{c}{ Parameter } & AUC & P \\
mFO (in male) & 0.24 & 0.11 \\
mFO (in female) & 0.6 & 0.65 \\
RAP & 0.64 & 0.23 \\
Shimmer & 0.7 & 0.09 \\
NHR & 0.67 & 0.15 \\
VTI & 0.73 & 0.06
\end{tabular}

mFo Average Fundamental Frequency; RAP Relative Average Perturbation; NHR Noise to Harmonic Ratio; VTI Voice Turbulence Index

Table 4. Combination of parameters with difference for ROC

\begin{tabular}{|c|c|c|c|c|c|}
\hline Parameter & AUC & $P$ & cutoff & SE & SP \\
\hline$V T I+R A P^{1)}$ & 0.75 & $0.04^{*}$ & 0.41 & 0.92 & 0.5 \\
\hline VTI + shimmer $\left.{ }^{2}\right)$ & 0.75 & $0.04^{*}$ & 0.49 & 0.92 & 0.6 \\
\hline $\mathrm{VTI}+\mathrm{NHR}^{3)}$ & 0.79 & $0.01^{*}$ & 0.51 & 0.92 & 0.7 \\
\hline $\mathrm{VTI}+\mathrm{mfO}(\text { in male })^{4)}$ & 0.96 & $0.005^{*}$ & 0.8 & 0.8 & 1 \\
\hline$V T I+m f O(\text { in female })^{5)}$ & 0.85 & 0.18 & 0.37 & 1 & 0.8 \\
\hline $\begin{array}{l}\mathrm{VTI}+\mathrm{RAP}+\text { shimmer }+\mathrm{NHR}+\mathrm{mF} \\
\left.\mathrm{O}(\text { in } \mathrm{male})^{6}\right)\end{array}$ & 1 & $0.002^{*}$ & 0.5 & 1 & \\
\hline $\begin{array}{l}V T I+R A P+\text { shimmer }+N H R+m F \\
0(\text { in female) })^{7)}\end{array}$ & 1 & $0.025^{*}$ & 0.5 & 1 & 1 \\
\hline
\end{tabular}

1) Analyzed by binary logistic regression, $R^{2}=0.329, P=0.039$

2) Analyzed by binary logistic regression, $R^{2}=0.343, P=0.034$

3) Analyzed by binary logistic regression, $R^{2}=0.319, P=0.044$

4) Analyzed by binary logistic regression, $R^{2}=0.799, P=0.002$

5) Analyzed by binary logistic regression, $R^{2}=0.230, P=0.477$

6) Analyzed by binary logistic regression, $R^{2}=1.000, P=0.006$

7) Analyzed by binary logistic regression, $R^{2}=1.000, P=0.002$ mFo Average Fundamental Frequency; RAP Relative Average Perturbation; NHR Noise to Harmonic Ratio; VTI Voice Turbulence Index, SE Sensitivity, SP Specificity
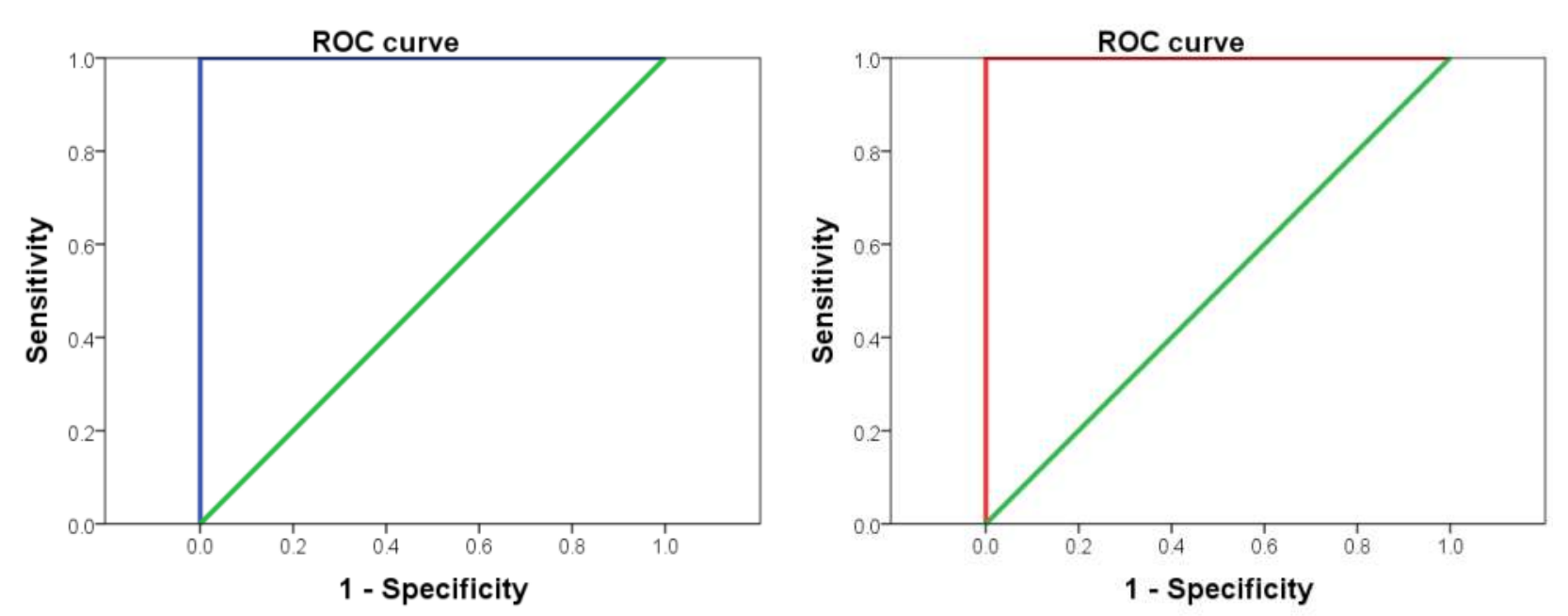

Figure 2. ROC curve explaining combination of all parameters for prediction of penestarion/ aspiration $(\mathrm{P} / \mathrm{A})$

Result

$>$ In the P/A group after VFSS, the values of $\mathrm{mFO}$ (in male), RAP, Shimmer, NHR were increasing, but the values of $\mathrm{mFO}$ (in female) and VTI were decreasing.

$>$ Significant change was noted in the VTI parameter between two groups $(p=0.028)$.

$>$ For both male and female, classification accuracy was $100 \%$ by enter method with five acoustic parameters

- Conclusion

$>$ Laryngeal aspiration influences acoustic voice parameters.

$>$ Especially, the change of VTI in P/A patients was statistically significant compared to that in Non-P/A patients.

> Acoustic voice analysis may be alternative evaluation for dysphagia. Also, the combination of several acoustic parameters may be screening tool for swallowing disorders. 